\title{
The IceCube-Gen2 High Energy Array
}

\section{The IceCube-Gen2 Collaboration ${ }^{\dagger}$}

${ }^{\dagger}$ http://icecube.wisc.edu/collaboration/authors/icrc15_gen2

E-mail: blaufuss a i cecube.umd.edu

Given recent observations of an astrophysical flux of neutrinos by the IceCube neutrino observatory, design studies for a next generation Antarctic neutrino observatory are well underway. The IceCube-Gen2 high-energy array will instrument a $\sim 10 \mathrm{~km}^{3}$ volume of clear glacial ice at the South Pole to deliver substantially larger astrophysical neutrino samples of all neutrino flavors. This detector would support a rich physics program, including a search for point sources, a detailed spectral and flavor characterization of the astrophysical neutrinos, searches for cosmogenic neutrinos, studies of cosmic rays, and searches for signatures of beyond-the-standard-model neutrino physics. This contribution highlights the detector sensitivity and geometry optimization studies performed during the design process. Astrophysical neutrino sensitivity can be increased by the addition of an extensive surface detector to identify and reject atmospheric backgrounds originating from the southern hemisphere. This large detector, in combination with the existing IceCube neutrino observatory and the rest of the IceCube-Gen 2 facility will be the flagship experiment of the new field of neutrino astronomy.

Corresponding authors: E. Blaufuss ${ }^{* 1}$, C. Kopper² ${ }^{2}$ C. Haack ${ }^{3}$,

${ }^{1}$ Department of Physics, University of Maryland, College Park, MD, USA

2 Department of Physics, CCIS 4-181, University of Alberta, Edmonton, Alberta T6G 2E1,

Canada

${ }^{3}$ III. Physikalisches Institut, RWTH Aachen University, D-52056 Aachen, Germany

The 34th International Cosmic Ray Conference,

30 July- 6 August, 2015

The Hague, The Netherlands

\footnotetext{
* Speaker.
} 


\section{Introduction}

High-energy neutrinos are unique in their ability to probe the extreme universe. Neutrinos reach the Earth from the edge of the universe without absorption or deflection by magnetic fields. They can escape from the deepest regions of cosmic accelerators where the highest energy cosmic rays are created. While these properties give them advantages over other astrophysical messengers such as photons and charged particles, their weak interactions make neutrinos very difficult to detect.

Detectors searching for these astrophysical neutrinos are built by instrumenting large natural liquid water or ice reservoirs to detect Cherenkov emission from the charged particles produced when the neutrinos interact with matter in or near the detector. The deep ice of the Antarctic glacier is host to IceCube [1], the first kilometer-scale neutrino observatory, which has recently reported an observed astrophysical neutrino signal [2, 3, 4, 5]. Proposed next-generation deepwater-based detectors include KM3NeT [6] in the Mediterranean sea and GVD [7] in Lake Baikal, both providing a complementary view of the sky to IceCube.

For an underground detector such as IceCube, the primary background is downward-directed muons created in cosmicray interactions in the Earth's atmosphere. These atmospheric muons trigger IceCube at a rate of $\sim 3 \mathrm{kHz}$. Atmospheric neutrinos are collected at a rate of $\sim 300$ per day, have a mean energy of $\sim 1 \mathrm{TeV}$ and form an isotropic background in searches for astrophysical neutrinos. At energies in excess of $\sim 100 \mathrm{TeV}$, the flux of atmospheric neutrinos is small, and upward-directed events of higher energy are likely of astrophysical origin.

A robust method to identify neutrino events is to distinguish events that start inside the detector from those which enter from the outside, which can be done for neutrino events originating from the entire sky. A search using this veto technique has been successfully applied to 4 years of data from the IceCube detector $[2,8]$. The 54 events found with deposited energies ranging from

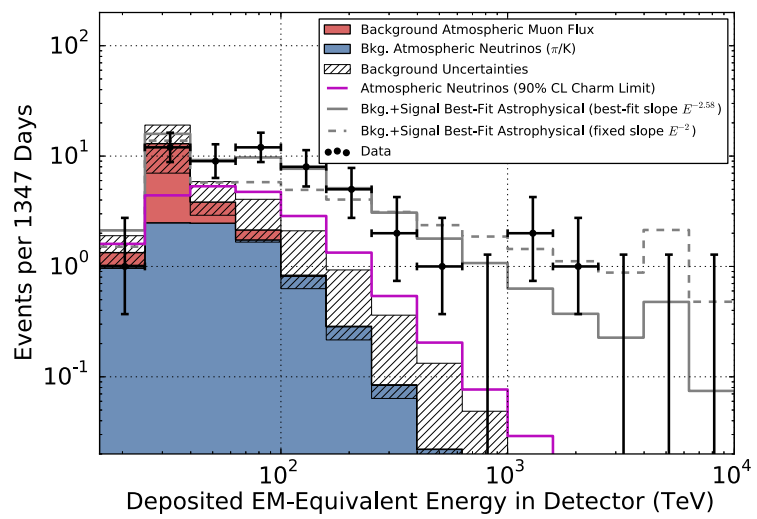

Figure 1: Deposited energies of contained events observed in 4 years of data in comparison to predictions. The hashed region shows uncertainties on the sum of all backgrounds. Muons (red) are computed from simulation to overcome statistical limitations in our background measurement and scaled to match the total measured background rate. Atmospheric neutrinos and uncertainties thereon are derived from models of both the $\pi, K$ and charm components of the atmospheric spectrum that have been fitted to the observed data [2].

$30 \mathrm{TeV}$ to $2 \mathrm{PeV}$ clearly reflect a significant

observation of astrophysical neutrinos. The deposited energy distribution for this sample, along with expected backgrounds and fits to signal hypotheses, is shown in Figure 1. An independent analysis of the spectrum of upward going muons arising from neutrinos passing through the Earth has confirmed the existence of the astrophysical component [9]. 
These modest sample sizes of astrophysical neutrinos represent the "first light" in the field of high-energy neutrino astronomy. With the present statistics, these astrophysical neutrino samples appear consistent with an isotropic, diffuse flux of neutrinos equally distributed between the three neutrino flavors. Additionally, all searches for individual galactic and extragalactic source candidates $[10,11,12]$ have so far only resulted in upper limits.

The effectiveness of IceCube as a tool for neutrino astronomy over the next decade is constrained by the limited numbers of astrophysical neutrinos measured in the cubic kilometer array. In this paper we present a vision for the next-generation IceCube neutrino observatory, the IceCubeGen2 high energy array, which is an expanded array of light-sensing modules that instrument a $\sim 10 \mathrm{~km}^{3}$ volume for detection of high-energy neutrinos [13]. With its unprecedented sensitivity and improved angular resolution, this instrument will explore extreme energies ( $\mathrm{PeV}$-scale) and will collect high-statistics samples of astrophysical neutrinos of all flavors, enabling detailed spectral studies, significant point source detections and new discoveries.

\section{Science Motivation}

The observation of astrophysical neutrinos has raised as many questions as it has answered. These include searches for the origin of these events, a full characterization of the flux, spectrum and flavor composition of the neutrinos, searches for neutrinos arising from the GZK process, searches for signals in coordination with other observatories and messenger particles, and improved measurements of the cosmic ray flux that drives our primary backgrounds. A detector with a significant increase in instrumented volume would aim to answer these questions.

With no indication of a steady point source in the current data, IceCube is increasingly sensitive to rare transient events, where observation of a multiplet of events in a short time can yield a significant detection. Sensitivity gains to transient events grow quickly in a larger detector. A next-generation neutrino observatory with 5 times the point-source sensitivity of IceCube and otherwise similar detector performance is predicted to have an increased sensitivity to transient source densities and rates by about two orders of magnitude [14].

While spectral and flavor composition of the observed astrophysical neutrino events are consistent with a single power law and equal distributions between all neutrino flavors, better measurement of these quantities from an increased sample size would begin to probe the internals of the cosmic accelerators that generate these events. The neutrino production mechanisms $(p-p, p-\gamma$ interactions, or neutron decay) and details of magnetic fields in the source region all leave their imprint $[13,15]$ on the observed event sample. Additionally, galactic and extra-galactic components could be resolved independently.

Multi-messenger astronomy, the combination of observations of cosmic rays, neutrinos, photons of all wavelengths, and, in the near future, gravitational waves will represent a powerful opportunity to decipher the physical processes that govern the non-thermal universe. With its improved sensitivity, IceCube-Gen 2 will be a unique instrument to complement the next generation of telescopes. Neutrinos play a central role in multi-messenger astronomy, as they are an unambiguous signature for the acceleration and interaction of protons and nuclei. IceCube already has a longstanding coordinated observation programs with several instruments [16, 17, 18, 19]. 
The surface array IceTop [20] has proved to be a valuable component of IceCube. Accordingly, the Gen2 high-energy array would include a surface detector near the top of each deployed string. With a spacing of $\sim 250 \mathrm{~m}$, such a surface array will provide a high-resolution measurement of the primary spectrum from $10 \mathrm{PeV}$ to above one EeV. Most importantly, with the larger aspect ratio of the IceCube-Gen2 high-energy array the acceptance for coincident events seen by both the surface array and the deep array increases by a factor of 40 , from $0.26 \mathrm{~km}^{2} \mathrm{sr}$ to $\sim 10 \mathrm{~km}^{2} \mathrm{sr}$. The ratio of the signal of $\sim \mathrm{TeV}$ muons in the deep detector to the size of the surface shower will allow an unprecedented measurement of the evolution of the primary composition in the region where a transition from galactic to extragalactic cosmic rays is predicted [21].

Astrophysical neutrinos at high energies are separable from atmospheric neutrinos by searching for other particles produced in the extended air shower that gives rise to these backgrounds. If high-energy neutrinos were produced in atmospheric air showers, they would likely be accompanied in the in-ice detector by muons produced in the parent air shower from which they originate. For $1 \mathrm{PeV}$ down-going atmospheric neutrinos, where the expectation is a few events per year, this atmospheric in-ice self-veto would reject more than $99.9 \%$ of them by observing an accompanying muon in the deep detector in coincidence with the neutrino [22, 23]. Additionally, a surface array can also act as a veto for cosmic-ray and atmospheric neutrino backgrounds to high-energy neutrino searches in the deep detector. While IceTop provides a veto that covers only about 3\% of the southern sky, the larger aspect ratio of the high-energy array would provide veto coverage to approximately $20 \%$ of the southern sky. Strategies are under study to extend the surface array beyond the footprint of the array $[24,25]$.

\section{Design of a Gen2 High Energy Array}

IceCube-Gen 2 would build upon the existing IceCube detector infrastructure, and would take advantage of the very long absorption lengths found in the glacial ice at the South Pole to add additional instrumentation with significantly larger string separation distances [26]. Building this larger instrument would be achieved with a comparable number of strings used in the existing IceCube detector, and would target neutrino energies above $\sim 50 \mathrm{TeV}$ with high efficiency.

While detailed studies toward a final design are ongoing, several design considerations are clear and understood. A detector sensitive to high-energy astrophysical muon, electron and tau neutrino flavors requires the correct combination of instrumented volume and projected surface area in all directions. This optimization is done by selecting larger string spacings, which increase the energy threshold as the instrumented volume increases, and by the geometrical arrangement of the deployed strings, which can trade detector instrumented volume for detector cross-sectional area. Detection of neutrino-induced muon track events will scale with the detector cross-sectional area, while the neutrino shower events produced by electron and tau flavors and neutral current interactions will scale with instrumented volume. Angular reconstruction performance, energy resolution and veto efficiency to astrophysical events are key metrics in the design study.

The most important aspect of a larger instrument in the glacial ice are the optical properties of the ice, in particular, the absorption length for Cherenkov photons. Typical absorption lengths are between $50 \mathrm{~m}$ and $200 \mathrm{~m}$ in the upper half of the detector, and often exceed $200 \mathrm{~m}$ in the lower half. Although the optical properties vary with the layered structure of the ice, the average absorption and 
scattering lengths dictate the distance by which one can space strings of sensors without impacting the uniform response of the detector. Early studies indicate that spacings of $\sim 240-300 \mathrm{~m}$ are acceptable while maintaining high efficiency to astrophysical neutrinos.

The optical properties of the glacial ice prevent us from using optical modules at depths much shallower than the current instrumented range used by IceCube (with instrumented depths between $1450 \mathrm{~m}$ and $2450 \mathrm{~m})$. Measurements of the depth dependence of the absorptivity of the Antarctic ice, Fig. 2, indicate that we will be able to extend the strings by $\sim 250 \mathrm{~m}$, leading to an increase in the geometric area for horizontal track events and therefore a $25 \%$ increase in effective area for such events.

To investigate the sensitivity of a larger detector, several benchmark geometries have been evaluated in software simulations, and are shown in Figure 3. These benchmark geometries use a non-regular grid pattern to avoid symmetries that deteriorate acceptance and resolution for muon tracks. They are compared to the IceCube detector in its completed 86 string configuration, and are used to scale sensitivities to a $10 \mathrm{~km}^{3}$ instrument. The projected areas of some of these geometries considered are compared to IceCube- 86

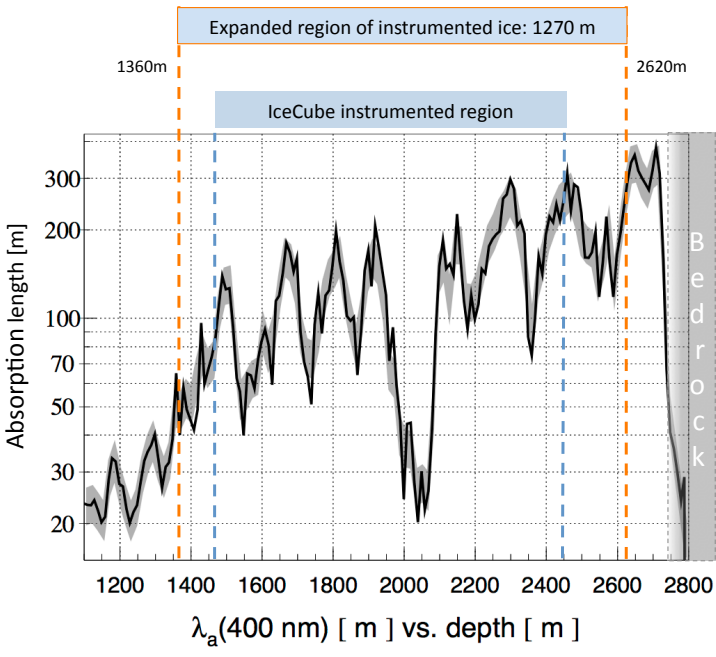

Figure 2: Absorption length in the glacial ice versus depth [26]. Note the layer of high dust concentration starting at about $2000 \mathrm{~m}$ depth. The ice above and below that layer is very clear. The current instrumented depth range used in IceCube and an extended string length, adding about $260 \mathrm{~m}$ to each string are indicated. Note that not all simulations shown in this report have been performed with the extended string length.

in Figure 4. As the detector volume grows in

these geometries, the exposed area increases and reaches up to $\sim 10 \mathrm{~km}^{2}$ area, substantially larger than the IceCube area.

For point source searches, which rely on muon tracks produced by charged current interactions of muon neutrinos in or near the instrumented volume, the sensitivity increases with the projected cross sectional area relative to source direction. At the energies of interest for astrophysical neutrino searches, these muons have ranges that routinely exceed $10 \mathrm{~km}$, greatly extending the sensitivity of these searches. Point source sensitivities will scale approximately with the square-root of the increase in cross sectional area and linearly with the improvement in angular resolution. In scenarios where the atmospheric backgrounds are negligible (e.g. short transients or searches for sources of very high-energy neutrinos), sensitivities are expected to scale nearly linearly with cross sectional area.

These benchmark geometries have been used to study the potential gains in point source sensitivity. Improvements in track angular resolution and overall point source sensitivity will be presented at the conference. 

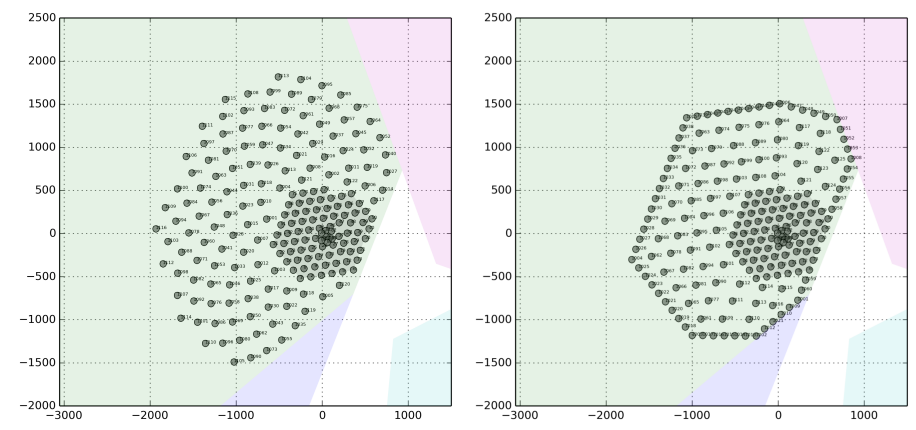

Figure 3: Example benchmark detector string layouts under study. Each expands about IceCube by adding 120 strings constrained to the South Pole "Dark Sector" (shaded in light green). For the left panel, uniform string spacing of $\sim 240 \mathrm{~m}$ is shown, and benchmark samples for $\sim 200 \mathrm{~m}$ and $\sim 300 \mathrm{~m}$ are also considered. The instrumented volumes are $6.0 \mathrm{~km}^{3}, 8.0 \mathrm{~km}^{3}$, and $11.9 \mathrm{~km}^{3}$ respectively. The panel on the right represents a string layout with a denser edge weighting, where edge strings are spaced at $\sim 125 \mathrm{~m}$, while interior strings are spaced at $\sim 240 \mathrm{~m}$, with an instrumented volume of $6.2 \mathrm{~km}^{3}$.

A search for electron or tau neutrino interactions depends on observation of an electromagnetic or hadronic cascade resulting from the interaction of the neutrino with nucleons inside the instrumented volume. These neutrinos generally interact with nucleons in the ice via deep-inelastic scattering processes, but at $E_{V} \sim 6.3 \mathrm{PeV}$ the resonant formation of an on-shell $W^{-}$-boson, the socalled Glashow resonance [27], enhances the the cross-section for electron-antineutrinos. The resonance would be observable mostly as a peak in the cascade energy spectrum, and serves as a key tag for neutrino flavor in a larger detector. Benchmark detectors show increased rates proportional to the volume gains, with factor of $\sim 10$ gains in observable Glashow resonance events. Given the similar event signatures for tau neutrinos, the same event rate gains in the larger detector are also found for tau neutrino events [13].

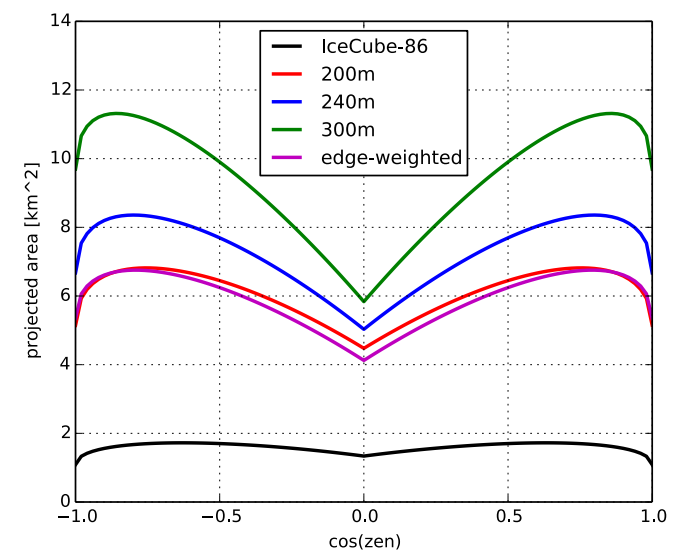

Figure 4: Instrumented area as a function of IceCube zenith angle for all benchmark configurations. To determine the geometric area, the detector volume containing all optical modules has been extended by $60 \mathrm{~m}$ on all sides. The figure legend also shows the instrumented volume of each detector, again with an extension of $60 \mathrm{~m}$ at the sides, top and bottom.

\section{Realization}

The proposed IceCube-Gen2 high-energy array is envisioned to be the major element of a planned large-scale enhancement to the IceCube facility at the South Pole station. Members of the IceCube-Gen2 Collaboration are working to develop proposals in the US and elsewhere that will 
include this next generation IceCube high-energy neutrino detector and the PINGU sub-array [28] that targets precision measurements of the atmospheric oscillation parameters and the determination of the neutrino mass hierarchy. The facility's reach may further be enhanced by exploiting the air-shower measurement and vetoing capabilities of an extended surface array [24, 25] and potentially a radio-pulse neutrino detector to achieve improved sensitivity to neutrinos in the $10^{16}$ $10^{20} \mathrm{eV}$ energy range, including GZK neutrinos.

A key for the successful scientific operation of IceCube has been the excellent reliability of the Digital Optical Modules (DOMs), hot-water drilling, DOM deployment operations [29] and data systems [30], which have resulted in stable data taking at more than $99 \%$ uptime. The instrumentation and drilling systems for IceCube-Gen2 closely follow the demonstrated IceCube technologies, with targeted improvements for overall performance and new capabilities. While the IceCube-Gen2 baseline DOM [31] keeps the robust structural elements of the IceCube DOM, a modern, more powerful set of electronic components are included into the design. In addition, DOM designs that utilize two large PMTs per DOM [32], multiple small PMTs per DOM [33], or wavelength-shifting and light-guiding techniques [34] are also being studied. The Enhanced Hot Water Drill design focuses on a modular system that will operate with higher efficiency and require less maintenance in routine operation. Both the instrumentation design (DOM, cable and readout) and deployment (EHWD) are shared with the PINGU low-energy array as part of the IceCube-Gen2 infrastructure, providing significant reductions in cost and enhancing flexibility.

\section{References}

[1] Ahrens, J., et al., Astropart.Phys. 20 (2004)

[2] IceCube Coll., Observation of Astrophysical Neutrinos in Four Years of IceCube Data, PoS(ICRC2015) 1081, These proceedings

[3] IceCube Coll., Update of a Combined Analysis of the High-Energy Cosmic Neutrino Flux at the IceCube Detector, PoS(ICRC2015) 1066, These proceedings

[4] IceCube Coll., A measurement of the diffuse astrophysical muon neutrino flux using multiple years of IceCube data, PoS(ICRC2015) 1079, These proceedings

[5] IceCube Coll., High energy astrophysical neutrino flux characteristics for neutrino-induced cascades using IC79 and IC86-string IceCube configurations, PoS(ICRC2015) 1109, These proceedings

[6] Margiotta, A., JINST 9 (2014) C04020

[7] Avrorin, A.D, et al., Nucl.Instrum.Meth. A742 (2014) 82

[8] Aarsten, M.G. et al., Phys.Rev.Lett. 113 (2014) 101101

[9] Aarsten, M.G. et al., Submitted to Phys.Rev.Lett.

[10] Aartsen, M.G. et al., Astrophys.J. 779 (2013) 132

[11] Adrian-Martinez, S. et al., Astrophys.J. 786 (2014) L5

[12] IceCube Coll., Results of neutrino point source searches with 2008-2014 IceCube data above $10 \mathrm{TeV}$, PoS(ICRC2015) 1047, These proceedings

[13] Aartsen, M.G. et al., IceCube-Gen2: A Vision for the Future of Neutrino Astronomy in Antarctica, [arxiv:1412.5106] 
[14] Ahlers, Markus and Halzen, Francis, Pinpointing Extragalactic Neutrino Sources in Light of Recent IceCube Observations, [arxiv:1406.2160]

[15] Kashti, Tamar and Waxman, Eli, Phys.Rev.Lett 95 (2005) 181101

[16] IceCube Coll., The Online Follow-Up Framework for Neutrino-Triggered Alerts from IceCube, PoS(ICRC2015) 1069, These proceedings

[17] IceCube Coll., Neutrino-triggered target-of-opportunity programs in IceCube, PoS(ICRC2015) 1052, These proceedings

[18] IceCube Coll. et al., Correlation between the UHECRs measured by the Pierre Auger Observatory and Telescope Array and neutrino candidate events from IceCube, PoS(ICRC2015) 1082, These proceedings

[19] M. Santander et al., Searching for TeV gamma-ray emission associated with IceCube high-energy neutrinos using VERITAS, PoS(ICRC2015) 785, These proceedings

[20] Aartsen, M.G. et al., Phys.Rev D88 (2013) 042004

[21] IceCube Coll., Cosmic Ray Science Potential for an Extended Surface array at the IceCube Observatory, PoS(ICRC2015) 694, These proceedings

[22] Schönert, Stefan and Gaisser, Thomas K. and Resconi, Elisa and Schulz, Olaf, Phys.Rev. D79 (2009) 043009

[23] Gaisser, Thomas K. and Jero, Kyle and Karle, Albrecht and van Santen, Jakob, Phys.Rev. D90 (2014) 023009

[24] IceCube Coll., Simulation Studies for a Surface Veto Array to Identify Astrophysical Neutrinos at the South Pole, PoS(ICRC2015) 1070, These proceedings

[25] IceCube Coll., Motivations and Techniques of a Surface Detector to Veto Air Showers for Neutrino Astronomy with IceCube at the Southern Sky, PoS(ICRC2015) 1156, These proceedings

[26] Aartsen, M.G. et al., Nucl. Instr. Meth. A711 (2013) 73

[27] Glashow, Sheldon L, Phys.Rev. 118 (1960) 316

[28] IceCube Coll., Status of the PINGU Detector PoS(ICRC2015) 1174, These proceedings

[29] Abbasi, R. et al., Nucl.Instrum.Meth. A618 (2010) 139

[30] Abbasi, R. et al.,Nucl.Instrum.Meth. A601 294

[31] IceCube Coll., Generation 2 IceCube Digital Optical Module and DAQ, PoS(ICRC2015) 1148, These proceedings

[32] IceCube Coll., A dual-PMT optical module (D-Egg) for IceCube-Gen2, PoS(ICRC2015) 1137, These proceedings

[33] IceCube Coll., Multi-PMT optical modules for IceCube-Gen2, PoS(ICRC2015) 1147, These proceedings

[34] D.Hebecker et al., Progress on the Development of a Wavelength-shifting Optical Module, PoS(ICRC2015) 1134, These proceedings 